Title: The influence of exercise intensity and exercise mode on gastrointestinal damage

Kate H. Edwards ${ }^{1}$, Kiran D. Ahuja ${ }^{1}$, Greig Watson ${ }^{1}$, Courtney Dowling ${ }^{1}$, Harrison Musgrave ${ }^{1}$, Jessica Reyes $^{1}$, James Cherry ${ }^{1}$, Cecilia M. Kitic ${ }^{1}$

1. Sports Performance Optimisation Research Team, School of Health Sciences, College of Health and Medicine, University of Tasmania

Corresponding Author

Kate H. Edwards

kate.edwards0@utas.edu.au

Sports Performance Optimisation Research Team, School of Health Sciences, College of Health and Medicine, University of Tasmania, Locked Bag 1322, Newnham, Launceston, Tasmania, 7250 


\begin{abstract}
Strenuous exercise increases gastrointestinal damage, but the dose-response relationship is yet to be elucidated. It is also commonly believed that running causes greater gastrointestinal damage than cycling. Two randomised, cross-over studies aimed to 1) quantify gastrointestinal damage with increasing exercise intensity, and 2) determine if running was associated with greater gastrointestinal damage than cycling. Following a $\mathrm{VO}_{2 \max }$ test, participants completed three cycling trials at different intensities ( $60 \mathrm{~min}$ at $40 \%, 60 \%$ and $80 \% \mathrm{VO}_{2 \max } ; n=10$ ( 5 female, 5 male)) (INTENSITY), or one running and one cycling trial ( $45 \mathrm{~min}$ at $70 \% \mathrm{VO}_{2 \max } ; n=11$ (3 female, 8 male)) (MODE). Venous blood samples were collected pre- and post- exercise to measure gastrointestinal damage via intestinal fatty acid binding protein (I-FABP). In INTENSITY, I-FABP magnitude of change was greater at $80 \% \mathrm{VO}_{2 \max }$ than $40 \% \mathrm{VO}_{2 \max }(p<0.01)$. In MODE, I-FABP magnitude of change was greater with cycling (mean (SD)) (84.7 (133.2)\% $d=1.07)$ compared to running $(19.3(33.1) \%, d=0.65)$ with a moderate effect $(d=0.68, p=0.024)$. RPE and HR were higher during cycling (RPE $p<0.0001 ; \mathrm{HR}$ $p<0.0001)$ but rectal temperature was not different between modes $(p=0.94)$. While gastrointestinal damage increases with increasing exercise intensity, running was not associated with greater gastrointestinal damage than cycling.
\end{abstract}

\title{
Novelty Bullets
}

- A fraction of the anaerobic threshold, rather than a fraction of $\mathrm{VO}_{2 \max }$, may be more predictive of intensity that results in exercise induced gastrointestinal damage

- The mode of exercise may not be as important as intensity for inducing gastrointestinal damage

- Improving anaerobic threshold may reduce susceptibility to gastrointestinal damage when exercising at high intensities

Keywords: running, cycling, I-FABP, gastrointestinal symptoms, intestinal permeability, threshold 


\section{Introduction}

Strenuous exercise impacts on the integrity and function of the gastrointestinal (GI) tract, the local and systemic consequences of which are described in a model of "Exercise-Induced Gastrointestinal Syndrome" (EIGS) (Costa et al. 2017). While the aetiology of EIGS is multifactorial, a primary factor is thought to be intestinal hypoperfusion and ischemia (de Oliveira \& Burini 2009). Briefly, as intensity increases, the redistribution of blood flow away from the gut toward working muscles results in an increasingly acidic and hypoxic environment (Van Wijck et al. 2011). As the intestinal mucosa becomes inflamed and enterocytes are damaged, gut integrity is compromised increasing gut permeability and potentially triggering endotoxemia (Costa et al. 2017). This disruption can result in an increased risk of infection, nutrient malabsorption and reduced fluid uptake leading to deleterious consequences for athletes in endurance events (Costa et al. 2017; van Wijck et al. 2013).

Endurance athletes will commonly report symptoms of EIGS such as diarrhoea, intestinal pain, nausea and vomiting during events (Peters et al. 1999; Pfeiffer et al. 2012; Stuempfle and Hoffman 2015) which are often a cause of event withdrawal/non-completion (Hoffman and Fogard 2011). Many symptoms are transient and benign, but some may be of greater medical consequence if prolonged (such as vomiting and diarrhoea), or chronic (such as systemic inflammation). Furthermore, substantial changes to gut integrity and function may be evident in asymptomatic athletes (Karhu et al. 2017), and may also occur after shorter events or training sessions, potentially impairing post-exercise recovery (van Wijck et al. 2013), negatively affecting subsequent exercise bouts and impacting adaptations to training.

Despite strenuous exercise being known to induce intestinal disruption, the intensity at which exercise is more likely to cause damage is yet to be fully elucidated as data on the dose-response relationship are scarce. Using a fraction of $\mathrm{VO}_{2 \max }$ as a measure of intensity is common within the 
literature related to exercise and intestinal damage, however methodological heterogeneity related to duration of exercise, environmental conditions, the fraction of $\mathrm{VO}_{2 \max }$ utilized and the method of determining gut disruption make comparisons difficult. Circulating intestinal fatty acid binding protein (I-FABP) in plasma has been identified as a sensitive and specific marker of damage to enterocytes (Ogden et al. 2020; Shi et al. 2015) as it is rapidly released into circulation upon intestinal mucosal injury and is highly correlated with ischemia (Shi et al. 2015). The temporal advantages of collection over more traditional methods such as use of a sugar probe (Ogden et al. 2020) allow for more immediate identification of intestinal disruption due to exercise. In thermoneutral, normobaric and normoxic conditions, increases in intestinal permeability (using a sugar probe) and/or I-FABP concentrations have been detected after as little as 20 minutes running at $80 \% \mathrm{VO}_{2 \max }$ (March et al. 2017), cycling for 60 minutes at $70 \% \mathrm{VO}_{2 \max }$ (Van Wijck et al. 2011) and running for 45 minutes at $70 \% \mathrm{VO}_{2 \max }$ (Lambert et al. 2008). Conversely, Hill et al. (2020) saw no significant change in I-FABP after running for 60 minutes at $65 \% \mathrm{VO}_{2 \max }$. Pals et al. (1997) observed that intestinal permeability increased at higher fractions of $\mathrm{VO}_{2 \text { peak }}$ when running for an hour $(80 \%$ of $\left.\mathrm{VO}_{2 \text { peak }}\right)$ but also noted a lack of elevated permeability at lower intensities ( $40 \%$ and $60 \% \mathrm{VO}_{2 \text { peak }}$ ) and suggested there is a potential threshold of exercise intensity at which an increase in intestinal permeability starts to occur. However, the sample size in Pals et al. was small $(n=6)$ and to date no other studies have investigated incremental changes in exercise intensity and markers of intestinal damage or function.

The mode of exercise may also impact susceptibility to intestinal damage as symptoms associated with EIGS tend to be reported with greater frequency and severity during running compared to other modes of exercise such as cycling, weightlifting and swimming (Clark et al. 1989; Peters et al. 1999; van Nieuwenhoven et al. 2004). Evidence that abdominal vibration is greater during running than other modes of exercise (Rehrer and Meijer 1991) has led to speculation that running creates greater mechanical stress (intra-abdominal pressure and movement of organs) and thus contributes 
to greater symptomology and intestinal damage (de Oliveira and Burini 2009; Stuempfle and Hoffman 2015; van Nieuwenhoven et al. 2004). However differences in gut dysfunction between modes of exercise have not been widely studied and, importantly, within single mode experimental studies, correlations are not always found between reported symptoms and physiological damage (Karhu et al. 2017).

Understanding the probability of intestinal damage occurring across increasing submaximal intensities, as well as the potential for some exercise modes to result in greater damage than others, may be a significant factor in interpreting the physiological impact of training loads for athletes. This may be especially important for multisport athletes, or athletes who regularly cross-train in different disciplines and who therefore may be more at risk of gut dysfunction following specific training sessions. Therefore, two studies were undertaken with the following aims: 1) to quantify intestinal damage with increasing exercise intensity, and 2) to determine if running is associated with greater intestinal damage than cycling when exercising at the same absolute $\mathrm{VO}_{2}$.

\section{Materials and Methods}

\section{Ethics}

The studies were approved by the Tasmania Health and Medical Human Research Ethics Committee (H0015313 and H0018160) and were performed in accordance with the standards set by the Declaration of Helsinki. The study protocols were explained in full to all participants through both written and oral format and all participants provided their written, informed consent.

\section{Recruitment}

Participants were recruited through social media and word of mouth. Healthy, moderately to welltrained individuals ( $>4$ hours training per week) aged 18-40 years old were eligible for recruitment. 
Exclusion criteria were current musculoskeletal injury and a history of medically diagnosed gastrointestinal disease (Crohn's, coeliac, ulcerative colitis, or other inflammatory bowel diseases). Females completed all trials in the self-reported luteal phase of their menstrual cycle. Participant characteristics are summarised in Table 1. One participant took part in both studies.

Assuming post-exercise I-FABP concentrations of $\sim 850 \pm 200 \mathrm{pg} \cdot \mathrm{mL}^{-1}$ (Pugh et al. 2017; Sheahen et al. 2018) and being able to detect a difference of $20 \%$ in response to different exercise modes and intensities, and a standard alpha (0.05) and beta value (0.8), a sample size of $n=11$ was calculated for each study.

\section{Design Overview}

The "INTENSITY" study measured changes in I-FABP after 60 mins of exercise at three different intensities (40, 60 and $80 \%$ of $\left.\mathrm{VO}_{2 \max }\right)($ Pals et al. 1997). The "MODE" study measured changes in IFABP after 45 minutes of running and cycling at the same absolute $\mathrm{VO}_{2 .}(70 \%$ cycle ergometer $\left.\mathrm{VO}_{2 \max }\right)$. Participants were randomised to the order in which they completed the trials using an online computer randomisation (sealedenvelope.com) and all participants completed all conditions within their study.

Participants visited the Human Performance Laboratory at the University of Tasmania either 4 times (INTENSITY) or 3 times (MODE). During the first visit, all participants completed an incremental maximal exercise test on a cycle ergometer which determined the target $\mathrm{VO}_{2}$ for the subsequent visits which were constant load trials specific to each study. Each visit was no earlier than 48 hours and no later than 7 days from the preceding visit and all trials were held at the same time of day $( \pm 1$ hour) for each participant. The study protocol is outlined in Figure 1.

**** Figure 1 about here **** 


\section{Incremental Maximal Exercise Test}

An incremental exercise test was used to determine maximal oxygen uptake $\left(\mathrm{VO}_{2 \max }\right)$ and target $\mathrm{VO}_{2}$. All tests were conducted in environmental conditions of $21^{\circ} \mathrm{C}$ and $40 \%$ relative humidity and performed on a Lode Cycle Ergometer (Lode, Groningen, Netherlands). Initial workload (measured in watts (W)) was $100 \mathrm{~W}$ for males and $75 \mathrm{~W}$ for females with an increase of $20 \mathrm{~W}$ per minute for males and $15 \mathrm{~W}$ per minute for females. Expired air was collected at 15 second intervals using a metabolic cart (Parvo TrueOne; Parvomedics, UT, USA). Heart rate (HR) (Polar FT1, Electro Oy, Kempele, Finland), power output and rating of perceived exertion (RPE) (6-20 Borg scale) (Borg 1982) were recorded at each minute interval. Criteria for attainment of $\mathrm{VO}_{2 \max }$ included three of the following: RER $>1.1$, HR within 10 beats of age predicted max, a plateau in oxygen uptake despite an increase in workload and/or volitional fatigue.

\section{INTENSITY Study}

Participants were instructed to refrain from strenuous exercise for 24 hours preceding each trial and from eating any food and drinking caffeine 2 hours before testing. Participants completed a food diary to record all food and fluid consumed in the 24 hours prior the first trial and were required to replicate their dietary intake for each subsequent trial. Upon arrival at the laboratory participants provided a pre-exercise venous blood sample and were provided with a 9FR rectal temperature probe (Phillips Medical Systems, MA, USA) for self-insertion $15 \mathrm{~cm}$ beyond the anal sphincter. A heart rate monitor strap (Polar Electro Oy, Kempele, Finland) was then fitted and body mass measured.

Participants completed a 5 minute warm up at $100 \mathrm{~W}$ for males and $75 \mathrm{~W}$ for females before commencing the 60 minute constant load exercise trial. $\mathrm{VO}_{2} \mathrm{~mL} \cdot \mathrm{kg}^{-1} \cdot \mathrm{min}^{-1}$, heart rate, RPE, self- 
reported GI symptoms (adapted from Pfeiffer et al. (2009)) and thermal comfort via the thermal sensation scale (TSS) (Gagge et al. 1967) were recorded every 5 minutes throughout the 60 minute trial. Rectal temperature was recorded every second for the duration of the exercise trial (EL-USBTP-LCD temperature data logger, Lascar, PA, USA). Every 5 minutes participants received a standardised bolus of water of $0.5 / 0.75 / 1.0 \mathrm{~mL} \cdot \mathrm{kg}^{-1} \cdot \mathrm{min}^{-1}$ for the $40 / 60 / 80 \%$ trials respectively. If $\mathrm{VO}_{2}$ varied from the prescribed exercise intensity $\left( \pm 2 \mathrm{~mL} \cdot \mathrm{kg}^{-1} \cdot \mathrm{min}^{-1}\right)$ power output was adjusted accordingly (increased or decreased) and expired air measured until two steady state readings at the target $\mathrm{VO}_{2}$ were achieved. Immediately upon completion of the exercise trial, participants provided a post-exercise venous blood sample, were re-weighed and then performed a 5 minute cool down before removing the HR monitor and rectal thermistor. Unfortunately, the COVID-19 pandemic prevented the recruitment of the final participant in the INTENSITY study and therefore $n=10$.

\section{MODE Study}

The protocol for the MODE study was nearly identical to the intensity study with 2 exceptions; 1 ) exercise time was 45 minutes instead of 60 minutes, and 2) the standard bolus of water was 1.25 $\mathrm{mL} \cdot \mathrm{kg}^{-1} \cdot \mathrm{min}^{-1}$ for both trials. The 45 minute exercise time was chosen as a duration that would elicit damage and enable comparison between running and cycling.

\section{Blood Collection and Analysis}

Venous blood samples were drawn without stasis by venepuncture from the antecubital fossa immediately pre- and post-exercise from participants in a supine position and collected into $6 \mathrm{~mL}$ Lithium Heparin Vacutainers ${ }^{\circledR}$ (Becton Dickinson, New Jersey, USA). A portion of whole blood was used immediately to take duplicate measures of haematocrit and haemoglobin (HemoCue ${ }^{\circledR} \mathrm{Hb} 20$ (HemoCue ${ }^{\circledR}$, Angelholm, Switzerland) to determine changes in plasma volume. Remaining blood was centrifuged at $4^{\circ} \mathrm{C}$ for 15 minutes at $1000 \mathrm{~g}$ and plasma aliquoted and stored at $-20^{\circ} \mathrm{C}$ until analysis.

\section{Assessment of Gastrointestinal Symptoms}


Gastrointestinal symptoms were rated using a 10-point scale ranging from 0 ("no problem at all") to 9 ("the worst it has ever been"). The questions were subdivided into 3 sections: upper abdominal, lower abdominal and other symptoms (Pfeiffer et al. 2009).

\section{Assessment of Intestinal Damage}

Pre- and post-exercise plasma I-FABP was used as a marker of enterocyte damage. I-FABP concentrations were determined using a commercial enzyme-linked immunosorbent assay (Hycult Biotechnology, Uden, The Netherlands) according to manufacturer's instructions. Results were adjusted for changes in plasma volume (Dill and Costill, 1974) and are reported in both absolute and percentage changes. The coefficient of variation was 3.4\% between sample duplicates.

\section{Determination of the Second Ventilatory Threshold}

As the intensity of exercise defined as a percentage of $\mathrm{VO}_{2 \max }$ may not adequately account for individual training status, we assessed the potential of a threshold effect of damage as suggested by Pals et al. (1997). Data from the incremental maximal test were plotted using GraphPad Prism version 8 (GraphPad Software, La Jolla California, USA). The second ventilatory threshold (VT2) was determined by visual analysis of the breakpoint where there was an increase in both the ventilatory equivalent of carbon dioxide $\left(\mathrm{VE} / \mathrm{VCO}_{2}\right)$ and the ventilatory equivalent of oxygen $\left(\mathrm{VE} / \mathrm{VO}_{2}\right)$.

\section{Statistical Analysis}

A Shapiro-Wilk test was used to determine normality of distribution of all data. For normally distributed data, ANOVA detected differences across intensities and paired t-tests determined preto post- differences within and between trials. For data that was not normally distributed, the Friedman test with Dunn's post hoc determined differences within trials, and the Wilcoxon matchedpairs signed rank test detected differences between trials. Pearson's correlation was used to identify a relationship between VT2 and I-FABP.

Cohen's effect size $(d)$ was calculated and inferred as small (0.2), moderate (0.5) or large (0.8) (Cohen, 1988). All statistical analyses were performed using GraphPad Prism version 8.4.2 for Windows (GraphPad software, La Jolla California, USA). Acceptance for significance was set at $p \leq$ 
0.05. Data are presented as mean (SD) (for normally distributed data) or median (interquartile range) (non-normally distributed).

\title{
Results
}

\section{Participant Characteristics}

\author{
**** Table 1 about here ****
}

\section{INTENSITY Study}

\section{Intestinal Fatty Acid Binding Protein}

Pre-exercise absolute I-FABP levels were not different between groups (40\%, 651.1 (271.6), 60\% $594.3(382.4), 80 \% 598.0(305.4), p=0.84)$. An overall main effect of intensity $(p=0.02)$ and a time (pre/post) $\mathrm{x}$ intensity interaction $(p=0.004)$ were observed for I-FABP. Absolute plasma I-FABP levels increased significantly pre-post in the $80 \%$ trial (mean(SD)) (pre: 598.0 (305.4) vs post: 1397 (902.0) $\mathrm{pg} \cdot \mathrm{mL}^{-1}, p=0.023, d=1.16$ ) but not in the $60 \%$ (pre: $594.3(382.4)$ vs post: $798.1(427) \mathrm{pg} \cdot \mathrm{mL}^{-1}, p=$ $0.065, d=0.43$ ) or $40 \%$ trials (pre: $651.1(271.6)$ vs post: $605.8(313.8) \mathrm{pg} \cdot \mathrm{mL}^{-1}, p=0.109, d=0.22$ ). The magnitude of change (\% increase in I-FABP) was significantly larger in the $80 \%$ trial (median (IQR): $148.3(150.5) \%)$ compared to either the $40 \%(-16.6(-18.5) \%)$ or $60 \%$ trials $(5.50(110.3) \%)(p=$ 0.007 and $p=0.05$ respectively). There was no statistical difference in the magnitude of change in IFABP between the $40 \%$ and $60 \%$ trials $(p=0.08)$ (Figure 2). There was a positive relationship between the magnitude of change in I-FABP and exercise intensity as determined by the ventilatory threshold $\left(r^{2}=0.60, p<0.0001\right)$ (Figure 3).

\footnotetext{
**** Figure 2 about here $* * * *$
}

\footnotetext{
**** Figure 3 about here $* * * *$
} 


\section{Physiological and Gastrointestinal Measures}

Actual $\mathrm{VO}_{2}, \mathrm{HR}$, core temperature and RPE were significantly different between all trials, increasing in line with the intensity of the trial. Participants lost more weight and plasma volume after the $80 \%$ trial compared to the $40 \%$ trial ( $p=0.01 ; p=0.04$ respectively) (Table 2 ).

Four out of the ten participants reported gastrointestinal symptoms during the $40 \%$ trial, 6 reported symptoms during the $60 \%$ trial and 8 during the $80 \%$ trial. The most common symptoms were belching, bloating and the urge to defecate. The two participants who did not report symptoms during the $80 \%$ trial also did not report symptoms during the other two trials.

There was a greater range of symptoms at $80 \%$ ( 9 symptoms) compared to both the 40 and $60 \%$ trials (4 symptoms each). Overall, most symptoms were mild (0-3 on a 10 point scale where 3 was "very minor problems"). The most severe symptom reported was the urge to defecate. Mean GI scores were similar between the $40 \%$ and $60 \%$ trials ( $40 \%: 0.2(0.58) ; 60 \%: 0.24(0.60) ; p=0.88)$, but scores were higher in the $80 \%$ trial $(0.70(1.01))$ compared to either the 40 or $60 \%$ trials (both $p$ values $<0.0001)$.

**** Table 2 about here

\section{MODE Study}

\section{Intestinal Fatty Acid Binding Protein}

There was no difference between trials for absolute I-FABP concentrations: pre-exercise running mean (SD) 803.9 (296.3) pg. $\mathrm{mL}^{-1}$ vs cycling 719.4 (320) pg. $\mathrm{mL}^{-1}, p=0.75$; post- exercise running 947.6 (449) $\mathrm{pg} \cdot \mathrm{mL}^{-1}$ vs cycling 1166 (562), $p=0.66$. Within trials, the mean increase in absolute I-FABP following the cycling trial was significantly different from baseline $(p=0.027)$ but was not different following the running trial $(p=0.16)$. The magnitude of change in I-FABP (\% change) was also significantly higher following cycling $(+84.7 \%)$ compared to running $(+19.3 \%) ; p=0.024, d=0.68$ 
(Figure 4). When the outlier (> 2SD) was removed, the magnitude of change following the cycling trial was still significantly higher than running $(p=0.05)$

\footnotetext{
**** Figure 4 about here ${ }^{* * * *}$
}

\section{Physiological and Gastrointestinal Measures}

Heart rate, RPE and TSS were significantly higher during cycling while rectal temperature was not different between trials (Table 3 ). Gastrointestinal symptoms we reported by $45 \%$ of participants during the running trial and $27 \%$ during the cycling trial. Across both studies, 55\% of participants reported no symptoms at all. All symptoms that were reported were mild $(<3$ on a 10 point scale where 3 = "very minor problems").

\section{**** Table 3 about here}

\section{Discussion}

The aims of these two studies were a) to quantify intestinal damage in relation to intensity of exercise, and b) to determine if running is associated with greater intestinal damage than cycling when exercising at the same absolute $\mathrm{VO}_{2 .}$. Our results show that damage to the gastrointestinal barrier is greater with increasing exercise intensity. Unexpectedly, running did not induce greater evidence of GI damage, rather, we observed a greater magnitude of change in I-FABP after cycling.

Pals et al. (1997) identified evidence of increasing gastrointestinal permeability as running intensity increased from $40 \% \mathrm{VO}_{2 \max }$ to $80 \% \mathrm{VO}_{2 \max }$, but found that after exercising at $60 \% \mathrm{VO}_{2 \max }$ the percent lactulose/rhamnose recovered was lower than at $80 \%$, but not different from $40 \%$. In the INTENSITY study, following a protocol similar to that as described in Pals et al. (1997), we show a similar pattern whereby there was no post-exercise increase in I-FABP after 60 mins of cycling at $40 \% \mathrm{VO}_{2 \max }$, but I- 
FABP concentrations more than doubled after exercising at $80 \% \mathrm{VO}_{2 \max }$. Results at $60 \% \mathrm{VO}_{2 \max }$ showed the largest inter-individual variability with four participants demonstrating a substantial increase in I-FABP and six not different from baseline.

Individual variation in I-FABP change was large in both studies, which is consistent with previous findings (March et al. 2019; Pugh et al. 2017). This may be partly because intensity defined as a fraction of $\mathrm{VO}_{2 \max }$, is physiologically not the same for all individuals. When data was analysed in the INTENSITY study using the second ventilatory threshold (VT2) as a measure of intensity, the results showed a stronger relationship between intensity and intestinal damage. The VT2 is a critical point that represents the maximal amount of work an individual can sustain for a prolonged period of time and is highly correlated with endurance performance (Joyner 1991). This threshold may occur from between $70 \%$ to $95 \%$ of $\mathrm{VO}_{2 \max }$ in different individuals and higher fractional utilisation is a hallmark of well-trained athletes. Thus, defining exercise intensity as a percentage of $\mathrm{VO}_{2 \max }$ may not account for individual variation along the intensity spectrum. In this context, the two outliers in the $80 \%$ trial are of interest. Both participants were well trained with a high VT2 and at $80 \%$ of $\mathrm{VO}_{2 \max }$ were working at a similar effort as some participants at $60 \% \mathrm{VO}_{2 \max }$ and evidence of gastrointestinal damage was minimal. Conversely, at $80 \% \mathrm{VO}_{2 \max }$, four participants were at or over $100 \% \mathrm{VT} 2$ and exhibited the largest increases in I-FABP. These findings suggest that using a measure such as VT2 rather than $\mathrm{VO}_{2 \max }$ may provide greater information about an individual's damage threshold and be key in avoiding chronic damage, reduced recovery, and impaired performance.

We had hypothesised that running, compared to cycling, would induce greater changes in I-FABP, primarily due to mechanical stress. Unexpectedly, there was a greater magnitude of change in IFABP following the cycling trial. To our knowledge only one previous study has directly compared gastrointestinal disruption in running and cycling. Compared to rest, van Nieuwenhoven et al. (2004) reported greater intestinal permeability following running for 90 minutes at $70 \%$ of $W_{\max }$ than after 
cycling, and suggested that that running may result in greater gastrointestinal ischemia. However, unlike van Nieuwenhoven et al. (2004) in which a $\mathrm{VO}_{2 \max }$ test was conducted on both a treadmill and cycle ergometer and intensity set relative to maximal power output in each mode, the present study used absolute oxygen uptake equal to $70 \% \mathrm{VO}_{2 \max }$ determined from cycle ergometry for both trials. Although cyclists are able to achieve similar $\mathrm{VO}_{2 \max }$ results when tested on both a cycle ergometer and a treadmill, most elite athletes will reach a higher max during running (Millet et al. 2009). While participants were exercising at the same absolute oxygen uptake in both trials, the higher HR and RPE we observed during the cycling trial indicates a higher physiological intensity which may be attributable to a smaller muscle mass having to work harder for the same $\mathrm{VO}_{2}$. A limitation to this study is that we did not obtain participants' $\mathrm{VO}_{2 \max }$ from a treadmill test to determine any differences with cycling and nor did we measure lactate concentrations and to do so would add to our understanding of thresholds relative to $\mathrm{VO}_{2}$ between modes of exercise. Our results however indicate that when running and cycling at the same absolute oxygen intake, the mechanical stress of running does not cause greater intestinal damage. Rather, it is the physiological intensity of the exercise, rather than the mode per se, that is the greater determinant of intestinal damage. Gastrointestinal symptoms across both studies were mild. The highest scores were recorded for the urge to defecate which was potentially related to the rectal thermistor as symptoms resolved upon removal. During the MODE study, a greater number of symptoms were reported during running, but there was a greater I-FABP increase following cycling. Neither the INTENSITY or MODE studies showed a relationship between reported symptoms and changes to I-FABP. This adds weight to the argument that Gl symptoms and GI damage are not necessarily related or indeed causal (Karhu et al. 2017; Pugh et al. 2017) and highlights the complexity surrounding the factors contributing to gastrointestinal disturbances that may interfere with athlete performance.

Previous literature has shown that an elevation in body temperature is related to increased intestinal perturbations (Pals et al. 1997; Snipe et al. 2018). We saw a significant increase in rectal 
temperature across the three trials in the INTENSITY study similar to observations made by Pals et al. (1997) although the relationship between core temperature and changes in I-FABP was weak $\left(r^{2}=\right.$ 0.18). During the MODE study, we observed an increased thermal sensation, heart rate and RPE during cycling along with increased I-FABP without a corresponding rise in core body temperature. Taken together, our current findings support the findings of Sheahen et al. (2018) who suggest that although a higher core temperature may exacerbate intestinal disruption, it is not a driving factor of exercise induced intestinal damage.

In conclusion, we show that increasing exercise intensity is associated with greater intestinal damage and that the individual VT2 may be more predictive of the intensity that induces intestinal damage, when compared to fraction of $\mathrm{VO}_{2 \max }$. Furthermore, by using an acute and sensitive marker of intestinal damage (I-FABP) to compare the effects of running and cycling on gastrointestinal disruption we show that the mechanical stress of running does not appear to cause additional damage. Rather, it is the physiological intensity of the exercise rather than the mode of exercise per se that will be a greater contributor to GI dysfunction. Further studies should explore the potential for identifying the damage threshold in individuals and extend on these findings to understand how that threshold may be determined across different modes of exercise to inform training guidelines for endurance and multi-sport athletes.

\section{Competing interests}

The authors declare there are no competing interests.

\section{Funding}

This study received no external funding. 


\section{References}

Borg, G.A. 1982. Psychophysical bases of perceived exertion. Med. Sci. Sports Exerc. 14(5): 377-381. doi:10.1249/00005768-198205000-00012.

Clark, C.S., Kraus, B.B., Sinclair, J., and Castell, D.O. 1989. Gastroesophageal Reflux Induced by Exercise in Healthy Volunteers. JAMA 261(24): 3599-3601. doi:10.1001/jama.1989.03420240113036.

Costa, R.J.S., Snipe, R.M.J., Kitic, C.M., and Gibson, P.R. 2017. Systematic review: exercise-induced gastrointestinal syndrome - implications for health and intestinal disease. Aliment. Pharmacol. Ther. doi:10.1111/apt.14157.

de Oliveira, E.P., and Burini, R.C. 2009. The impact of physical exercise on the gastrointestinal tract. Curr. Opin. Clin. Nutr. Metab. Care 12(5): 533-538. doi:10.1097/MCO.0b013e32832e6776.

Gagge, A.P., Stolwijk, J., and Hardy, J. 1967. Comfort and thermal sensations and associated physiological responses at various ambient temperatures. Environ. Res. 1(1): 1-20.

doi:10.1016/0013-9351(67)90002-3.

Hill, G.W., Gillum, T.L., Lee, B.J., Romano, P.A., Schall, Z.J., Hamilton, A.M., et al. 2020. Prolonged treadmill running in normobaric hypoxia causes gastrointestinal barrier permeability and elevates circulating levels of pro- and anti-inflammatory cytokines. Appl. Physiol. Nutr. Metab. 45(4): 376386. doi:10.1139/apnm-2019-0378.

Hoffman, M.D., and Fogard, K. 2011. Factors related to successful completion of a 161-km ultramarathon. Int. J. Sports Physiol. Perform. 6(1): 25-37. doi:10.1123/ijspp.6.1.25.

Joyner, M.J. 1991. Modeling: optimal marathon performance on the basis of physiological factors. J Appl Physiol (1985) 70(2): 683-687. doi:10.1152/jappl.1991.70.2.683.

Karhu, E., Forsgård, R.A., Alanko, L., Alfthan, H., Pussinen, P., Hämäläinen, E., et al. 2017. Exercise and gastrointestinal symptoms: running-induced changes in intestinal permeability and markers of gastrointestinal function in asymptomatic and symptomatic runners. Eur. J. Appl. Physiol. 117(12): 2519-2526. doi:10.1007/s00421-017-3739-1.

Lambert, G.P., Lang, J., Bull, A., Pfeifer, P., Eckerson, J., Moore, G., et al. 2008. Fluid restriction during running increases GI permeability. Int. J. Sports Med. 29(03): 194-198. doi:10.1055/s-2007-965163.

March, D.S., Jones, A.W., Thatcher, R., and Davison, G. 2019. The effect of bovine colostrum supplementation on intestinal injury and circulating intestinal bacterial DNA following exercise in the heat. Eur. J. Nutr. 58(4): 1441-1451. doi:10.1007/s00394-018-1670-9.

March, D.S., Marchbank, T., Playford, R.J., Jones, A.W., Thatcher, R., and Davison, G. 2017. Intestinal fatty acid-binding protein and gut permeability responses to exercise. Eur. J. Appl. Physiol. 117(5): 931-941. doi:10.1007/s00421-017-3582-4.

Millet, G.P., Vleck, V.E., and Bentley, D.J. 2009. Physiological Differences Between Cycling and Running. Sports Med. 39(3): 179-206. doi:10.2165/00007256-200939030-00002.

Ogden, H.B., Fallowfield, J.L., Child, R.B., Davison, G., Fleming, S.C., Edinburgh, R.M., et al. 2020. Reliability of gastrointestinal barrier integrity and microbial translocation biomarkers at rest and following exertional heat stress. Physiological Reports 8(5): e14374-e14374. doi:10.14814/phy2.14374.

Pals, K.L., Chang, R.-T., Ryan, A.J., and Gisolfi, C.V. 1997. Effect of running intensity on intestinal permeability. J. Appl. Physiol. 82(2): 571-576. doi:10.1152/jappl.1997.82.2.571. 
Peters, H., Bos, M., Seebregts, L., Akkermans, L., van Berge Henegouwen, G., Bol, E., et al. 1999. Gastrointestinal symptoms in long-distance runners, cyclists, and triathletes: prevalence, medication, and etiology. Am. J. Gastroenterol. 94(6): 1570-1581. doi:10.1016/S00029270(99)00203-8.

Pfeiffer, B., Cotterill, A., Grathwohl, D., Stellingwerff, T., and Jeukendrup, A.E. 2009. The effect of carbohydrate gels on gastrointestinal tolerance during a 16-km run. Int. J. Sport Nutr. Exerc. Metab. 19(5): 485-503. doi:10.1123/ijsnem.19.5.485.

Pfeiffer, B., Stellingwerff, T., Hodgson, A.B., Randell, R., Pöttgen, K., Res, P., et al. 2012. Nutritional intake and gastrointestinal problems during competitive endurance events. Med. Sci. Sports Exerc. 44(2): 344-351. doi:10.1249/mss.0b013e31822dc809

Pugh, J.N., Impey, S.G., Doran, D.A., Fleming, S.C., Morton, J.P., and Close, G.L. 2017. Acute highintensity interval running increases markers of gastrointestinal damage and permeability but not gastrointestinal symptoms. Appl. Physiol. Nutr. Metab. 42(9): 941-947. doi:10.1139/apnm-20160646.

Rehrer, N.J., and Meijer, G.A. 1991. Biomechanical vibration of the abdominal region during running and bicycling. J. Sports Med. Phys. Fitness 31(2): 231-234.

Sheahen, B.L., Fell, J.W., Zadow, E.K., Hartley, T.F., and Kitic, C.M. 2018. Intestinal damage following short-duration exercise at the same relative intensity is similar in temperate and hot environments. Applied physiology, nutrition, and metabolism 43(12): 1314-1320. doi:10.1139/apnm-2018-0057.

Shi, H., Wu, B., Wan, J., Liu, W., and Su, B. 2015. The role of serum intestinal fatty acid binding protein levels and D-lactate levels in the diagnosis of acute intestinal ischemia. Clin. Res. Hepatol. Gastroenterol. 39(3): 373-378. doi:10.1016/j.clinre.2014.12.005.

Snipe, R.M.J., Khoo, A., Kitic, C.M., Gibson, P.R., and Costa, R.J.S. 2018. The impact of exertional-heat stress on gastrointestinal integrity, gastrointestinal symptoms, systemic endotoxin and cytokine profile. Eur. J. Appl. Physiol. 118(2): 389-400. doi:10.1007/s00421-017-3781-z.

Stuempfle, K.J., and Hoffman, M.D. 2015. Gastrointestinal distress is common during a 161-km ultramarathon. J. Sports Sci. 33(17): 1814-1821. doi:10.1080/02640414.2015.1012104.

van Nieuwenhoven, M.A., Brouns, F., and Brummer, R.-J.M. 2004. Gastrointestinal profile of symptomatic athletes at rest and during physical exercise. Eur. J. Appl. Physiol. 91(4): 429-434. doi:10.1007/s00421-003-1007-z.

Van Wijck, K., Lenaerts, K., Van Loon, L.J., Peters, W.H., Buurman, W.A., and Dejong, C.H. 2011. Exercise-induced splanchnic hypoperfusion results in gut dysfunction in healthy men. PLoS One 6(7): e22366. doi:10.1371/journal.pone.0022366.

van Wijck, K., Pennings, B., van Bijnen, A.A., Senden, J.M., Buurman, W.A., Dejong, C.H., et al. 2013. Dietary protein digestion and absorption are impaired during acute postexercise recovery in young men. Am. J. Physiol. Regul. Integr. Comp. Physiol. 304(5): R356-R361. 


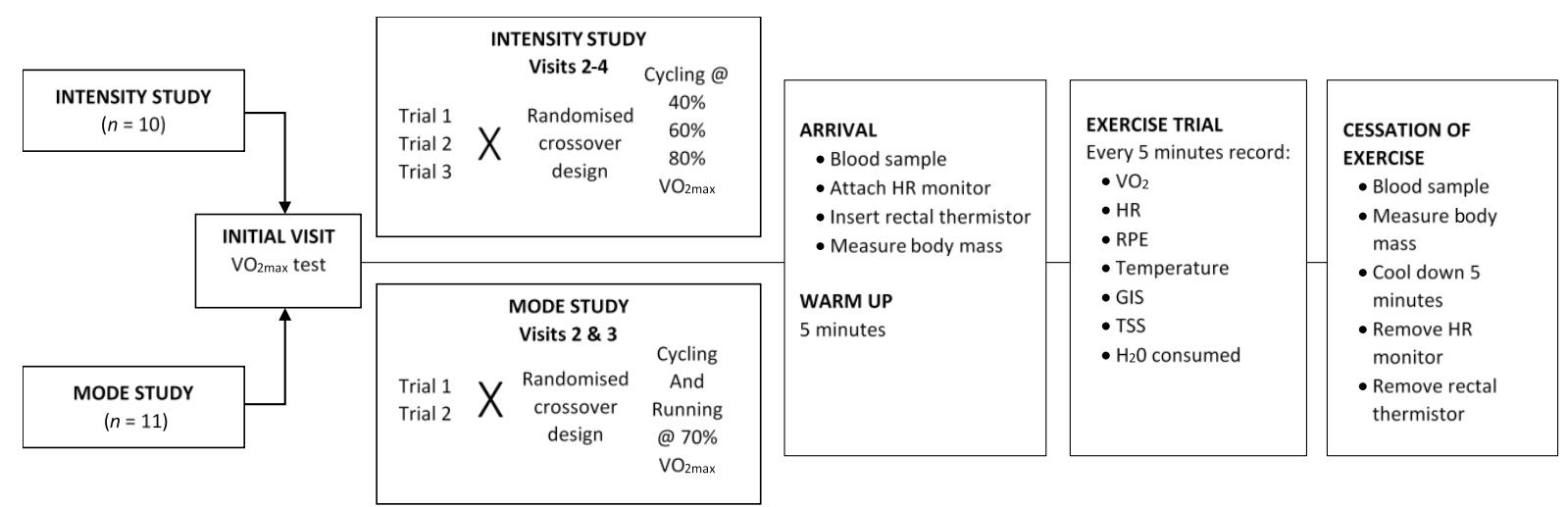

Fig. 1 Schematic overview of the experimental protocol ( VO2, volume of oxygen; HR, heart rate;

RPE, rating of perceived exertion; GIS, gastrointestinal symptoms; TSS, thermal sensation scale) 


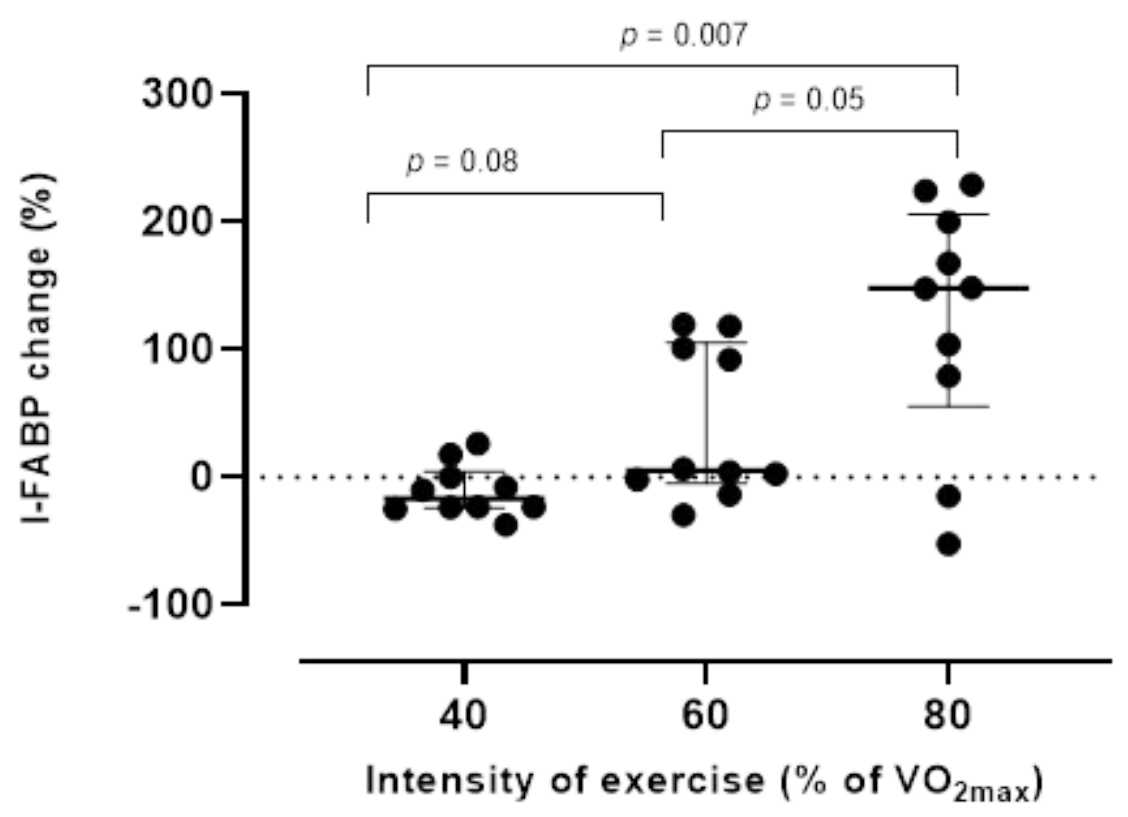

Fig. 2 Percentage change in plasma intestinal fatty acid binding protein (I-FABP) in response to 60 minutes of exercise at 40,60 and $80 \%$ of $\mathrm{VO}_{2 \max }(n=10)$. $\bullet$ indicate individual data points, bars indicate median and interquartile range. 


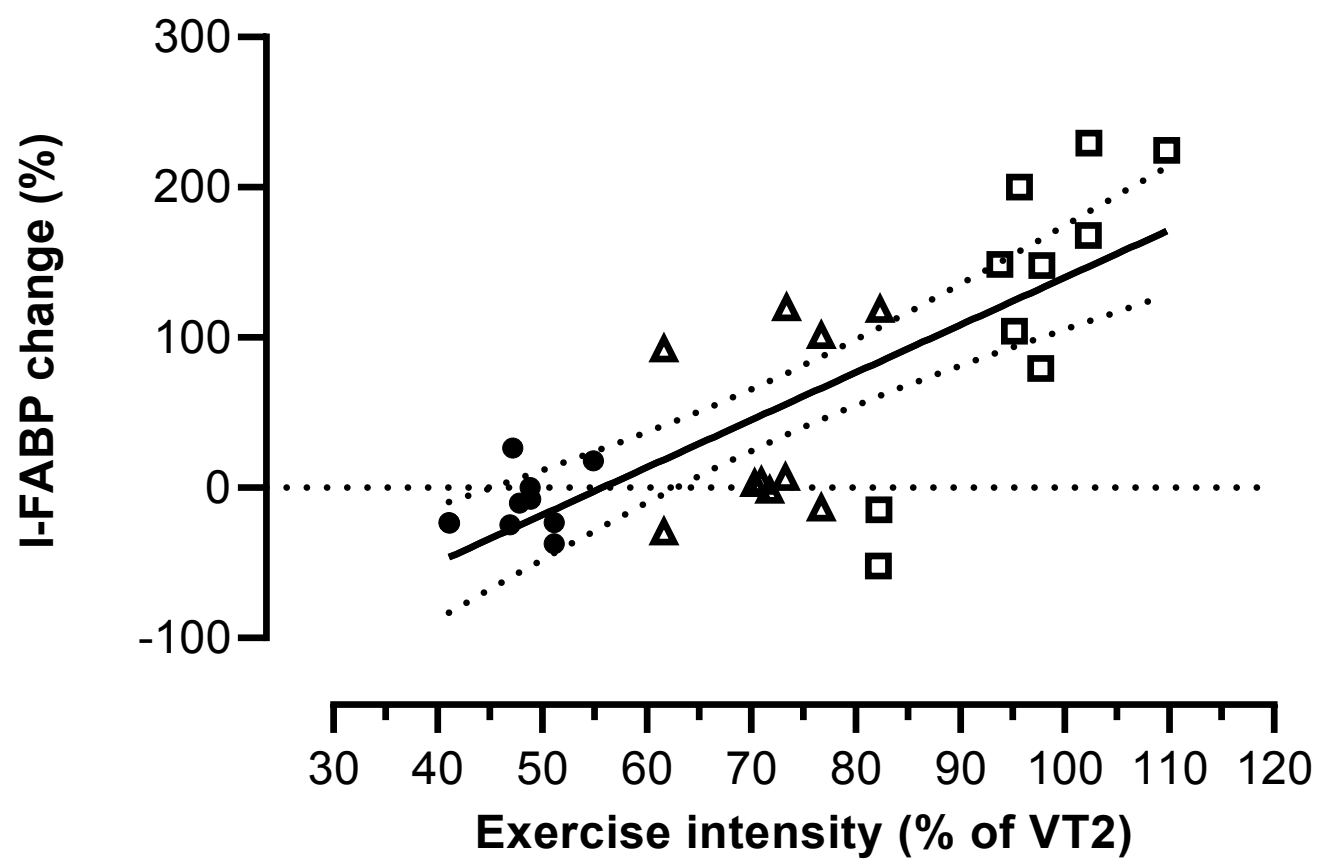

Fig. 3 Percentage change in plasma intestinal fatty acid binding protein (I-FABP) in relation to exercise intensity as determined by a fraction of the second ventilatory threshold (VT2) during the different trials $\left(\bullet 40 \%\right.$ trial; $\Delta 60 \%$ trial; $\square 80 \%$ trial ; $\left.r^{2}=0.60, p<0.0001\right)$. Dotted lines indicate $95 \%$ confidence intervals. 


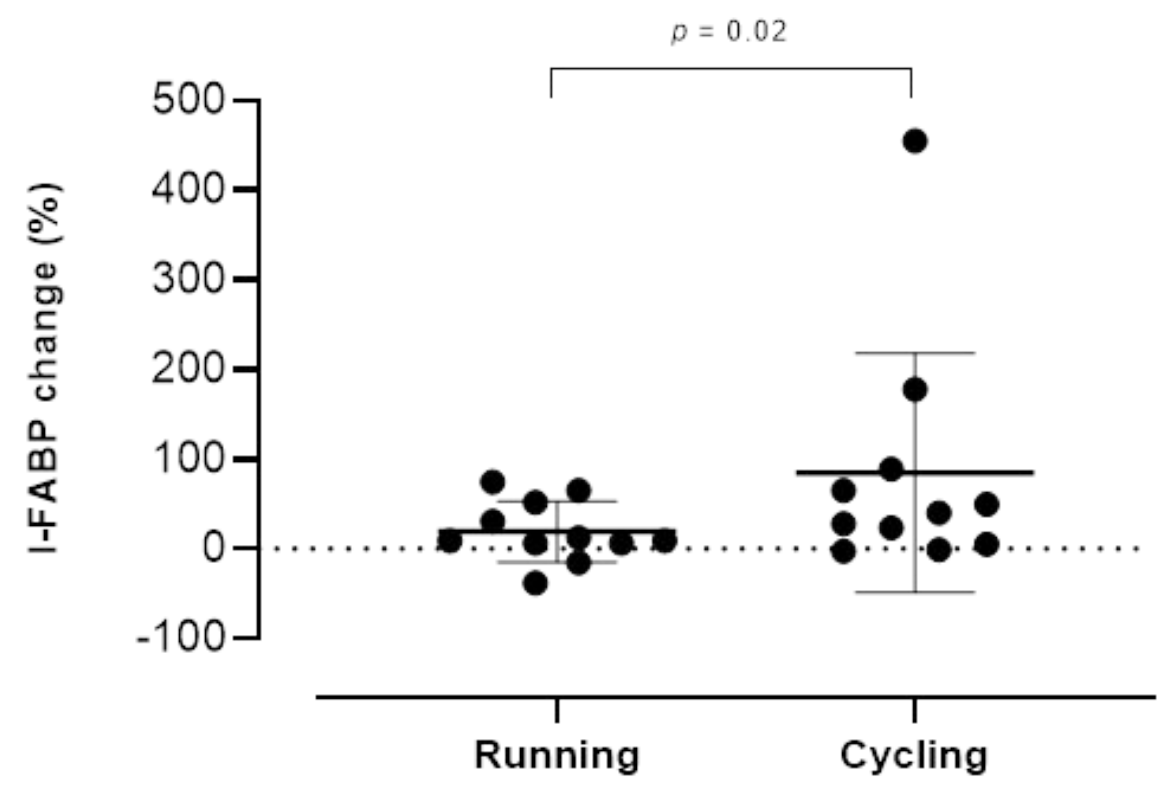

Fig. 4 Percentage change in plasma intestinal fatty acid binding protein (I-FABP) in response to 45 minutes of running and cycling at $70 \%$ of $\mathrm{VO}_{2 \max }(n=11)$. $\bullet$ indicate individual data points, bars indicate mean and standard deviation. 
Table 1 Participant characteristics

\begin{tabular}{lcc}
\hline & $\begin{array}{c}\text { Intensity Study } \\
\text { Mean (SD) }\end{array}$ & $\begin{array}{c}\text { Mode Study } \\
\text { Mean (SD) }\end{array}$ \\
\hline Participants & $n=10(5$ female; 5 male $)$ & $n=11$ (3 female; 8 male) \\
Age (years) & $27(7)$ & $25(7)$ \\
Body mass (kg) & $71.8(13.8)$ & $70.5(11.6)$ \\
Height (m) & $170.0(10.4)$ & $173.1(10.5)$ \\
BMI (kg.m $\left.{ }^{2}\right)$ & $24.6(2.1)$ & $23.4(2.5)$ \\
$\mathrm{VO}_{2 \mathrm{max}}\left(\mathrm{mL} \cdot \mathrm{kg}^{-1} \cdot \mathrm{min}^{-1}\right)$ & $46.0(6.8)$ & $51.6(5.3)$ \\
\hline
\end{tabular}


Table 2 Physiological and perceptual data during 60 minutes of exercise at different intensities

\begin{tabular}{|c|c|c|c|c|c|c|}
\hline & \multicolumn{3}{|c|}{$\begin{array}{l}\text { Intensity of Exercise } \\
\left(\% \mathrm{VO}_{2 \text { max }}\right)\end{array}$} & \multicolumn{3}{|c|}{$p$ values } \\
\hline & 40 & 60 & 80 & 40 vs 60 & 60 vs 80 & 40 vs 80 \\
\hline Actual $\mathrm{VO}_{2} \%$ & $41.3(1.6)$ & $61.3(1.5)$ & 79.5 (3.0) & $<0.0001$ & $<0.0001$ & $<0.0001$ \\
\hline Heart rate (bpm) & $115(13)$ & 145 (13) & $162(13)$ & $<0.0001$ & 0.0002 & $<0.0001$ \\
\hline Rectal temp $\left({ }^{\circ} \mathrm{C}\right)$ & $37.0(0.25)$ & $37.3(0.25)$ & $37.6(0.16)$ & 0.04 & 0.05 & 0.001 \\
\hline RPE & $10.1(1.5)$ & $13.0(1.7)$ & $16.0(1.5)$ & $<0.001$ & $<0.001$ & $<0.0001$ \\
\hline $\begin{array}{l}\text { Weight change } \\
\text { (kg) }\end{array}$ & $0.02(0.17)$ & $-0.09(0.15)$ & $-0.24(0.25)$ & 0.07 & 0.12 & 0.01 \\
\hline $\begin{array}{l}\text { Plasma volume } \\
\text { change } \%\end{array}$ & $-1.6(4.8)$ & $-3.5(5.3)$ & $-7.9(4.2)$ & 0.74 & 0.08 & 0.04 \\
\hline TSS & $0.8(0.13)$ & $1.5(0.29)$ & $2.1(0.38)$ & 0.04 & 0.04 & $<0.0001$ \\
\hline
\end{tabular}


Table 3 Physiological and perceptual data during 45 minutes of running and cycling.

\begin{tabular}{lccc}
\hline & Running & Cycling & $p$ values \\
\hline Actual $\mathrm{VO}_{\mathbf{2}} \%$ & $71.2(1.19)$ & $70.6(0.7)$ & 0.55 \\
Heart rate (bpm) & $153(11)$ & $165(12)$ & $\mathbf{0 . 0 0 0 1}$ \\
Rectal temp ( $\left.{ }^{\circ} \mathrm{C}\right)$ & $37.4(0.46)$ & $37.4(0.44)$ & 0.94 \\
RPE & $12.5(1.4)$ & $15.0(1.2)$ & $\mathbf{0 . 0 0 0 1}$ \\
Weight change (kg) & $-0.04(0.37)$ & $-0.25(0.31)$ & 0.13 \\
Plasma volume change \% & $0.6(4.8)$ & $-4.9(4.5)$ & $\mathbf{0 . 0 3 4}$ \\
TSS & $1.3(0.29)$ & $1.7(0.27)$ & $\mathbf{0 . 0 0 4}$ \\
\hline Data reported as mean (SD). RPE, rating of perceived exertion; TSS, thermal sensation scale $(n=11)$
\end{tabular}

